\title{
Superperconductors, Their History and Applications
}

\author{
Adem Beriso \\ Madda Walabu University, college of Natural and computational Science, Department of Physics, Bale-Robe, \\ Ethiopia; P.box: 247
}

\begin{abstract}
The phenomenon of zero resistance below certain low temperature (critical temperature) leads to limitless current which implies that no dissipation in electrical energy for this occurrence. This property is immediately followed by expulsion of magnetic field from the interior of a material if this material is placed in external magnetic while it is below this low temperature. Materials that have these unique properties when they are below their critical temperature are superconductor materials which were discovered in 1911 by famous Dutch physicist H. K. Onnes. In addition to the above mentioned properties, superconductors have many exceptional positive properties and thus have excellent potentials for applications in every sector. The main problem of these materials is their low temperature which needs refrigerator for their applications elsewhere which is too costly compared to conventional conductors. Even though having room temperature superconductors is still a dream, if it is achieved we can extract energy from these materials with very low cost. In this review history of superconductors, their type and some of their applications have been considered.
\end{abstract}

Keywords: Critical temperature; Superconductors; Zero resistance; Meissner effect; Limitless current

DOI: $10.7176 /$ APTA/77-02

Publication date:May $31^{\text {st }} 2019$

\section{INTRODUCTION}

Superconductors are the materials that have zero resistance at certain very low temperatures called critical (transition) temperatures, $T_{C}$ accompanied by a total expulsion of magnetic field from within (the Meissner effect)[1]. It was known that the electrical resistivity of metallic conductors is directly proportional to their temperature. Thus, as the temperature of a certain metallic conductors decreases their electrical resistivity decreases gradually and eventually drops suddenly to zero value at some critical (transition) temperature $T_{C}$, a phenomenon called superconductivity which was discovered in 1911 by the great Dutch physicist Heike Kamerlingh Onnes [2]. This phenomenon requires extremely low temperatures, generally somewhere just above absolute zero (0 degree Kelvin, -273 degree Celsius or -459 degree Fahrenheit). The value of the critical temperature $T_{C}$ at which materials have zero electrical resistance varies from one material to another material and it is achieved by cooling the materials with either liquid helium (for low temperature superconductors) or liquid nitrogen (for high temperature superconductors) [3][4].

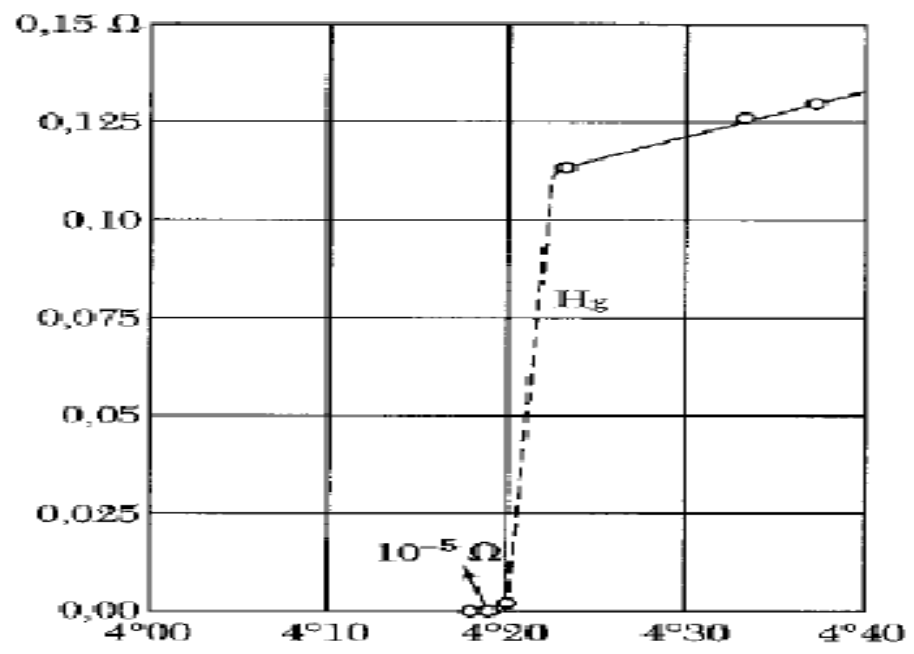

Figure 1: Sharp drop in resistance for mercury at $4.2 \mathrm{~K}[17]$. 
The Meissner Effect

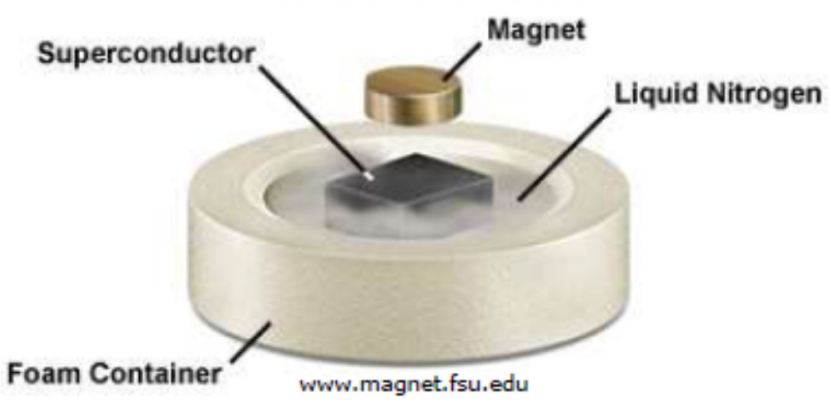

Figure 2:MeissnerEffect[5].

Now it is known that superconductivity occurs in wide variety of materials, including simple elements like aluminum and tin, various metallic alloys, some heavily-doped semiconductors and certain ceramic compounds containing planes of copper and oxygen atoms. The later classes of compounds, known as the cuprates, are high temperature superconductors. Superconductivity does not occur in noble metals like gold and silver, nor in ferromagnetic metals[6].

\section{Comparison between normal conductors and superconductors}

In normal conductors, an electrical current can be imagined as a fluid of electrons moving across a heavy ionic lattice. In such materials there is a constant collision between electrons and ions in the lattice, and during every one collision some of the energy carried by the current is absorbed by the lattice and converted into heat. As a result, the energy carried by the current is constantly being dissipated in the form of heat which leads to the phenomenon of electrical resistance. However, in superconductors the situation is different. In superconductors (especially type I superconductors), the electronic fluid cannot be resolved into individual electrons, instead consisting of bound pairs of electrons known as Cooper pairs. This pairing is caused by an attractive force between electrons from the exchange of phonons. Quantum mechanically, the energy spectrum of this Cooper pair fluid possesses an energy gap, meaning there is a minimum amount of energy $\Delta E$ that must be supplied in order to excite the fluid. Therefore, if $\Delta E$ is larger than the thermal energy of the lattice $(k T)$, where $\mathrm{k}$ is Boltzmann's constant and $\mathrm{T}$ is the temperature, the fluid will not be scattered by the lattice. The Cooper pair fluid is thus a superfluid, meaning it can flow without energy dissipation (with zero resistance)[7][8].

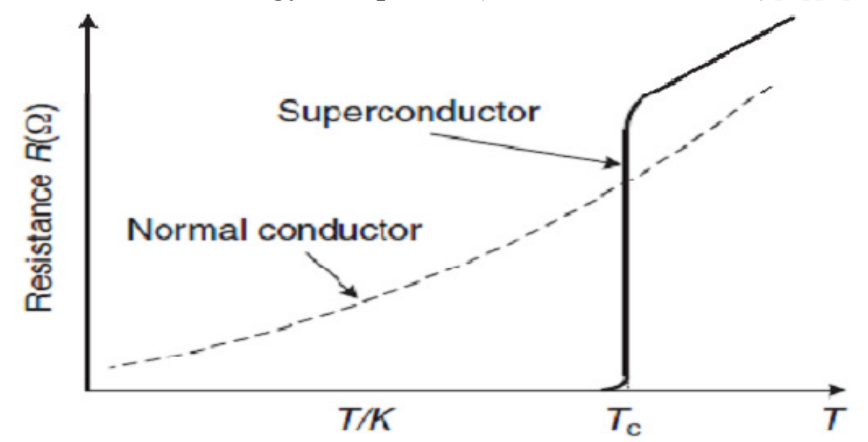

Figure 3: Resistance versus temperature curve of superconductors and normal conductors[2].

\section{HISTORICAL DEVELOPMENT}

The history of superconductors is very rich, consisting of many events and discoveries. Before the discovery of the phenomenon of superconductivity the value of lowest absolute temperature which is $-273{ }^{\circ} \mathrm{C}\left(0^{\circ} \mathrm{K}\right)$ was proposed in 1800 from the pressure-temperature (P-T) relationship by Charles and Gay-Lusac. Seventy seven years later, in 1877 Cailletet and Pictet liquefied Oxygen at $-183^{\circ} \mathrm{C}\left(90^{\circ} \mathrm{K}\right)$. Soon after, Nitrogen was liquefied at a temperature of $77^{\circ} \mathrm{K}$. In 1898 Dewar liquefied Hydrogen at $20^{\circ} \mathrm{K}[9]$.

On the $10^{\text {th }}$ July 1908 , in his laboratory at Leiden University, the great Dutch physicist Heike Kamerlingh Onnes (1853-1926) liquefied helium at $4.2^{\circ} \mathrm{K}$ and that was the day which opened an entirely new chapter in lowtemperature physics. Three years later, in 1911 Onnes with his team discovered Superconductivity in Holland while studying the electrical resistance of a sample of frozen mercury $(\mathrm{Hg})$ as a function of temperature. On cooling $\mathrm{Hg}$ to the temperature of liquid helium, he found that the resistance vanished abruptly at temperature approximately 4.2 K. This phenomenon was not completely explained at the time of discovery because the quantum theory of metal was not yet developed to its full potential in that days. In 1913 he won the Nobel Prize for the liquification of helium and the discovery of superconductivity. In subsequent decades, superconductivity was found in several 
other materials. In 1913, lead was found to be superconductor at 7K, and in 1941 niobium nitride was found to be superconductor at $16 \mathrm{~K}[10][11]$.

The next important step in understanding superconductivity occurred in 1933 by Walter Meissner and Robert Ochsenfeld when superconductors cooled below their critical temperatures, were placed in an external magnetic field, the magnetic flux were completely expelled from their interior, a phenomenon which is known as the Meissner effect. This technology has been practically used as mag-lev technology (magnetic levitation) that can power high speed trains, which was tested by Japan in 1999 for trains to have a high speed of 343 miles/hours. In this technology, strong superconducting magnets cause a transport vehicle to float above a track, thereby excluding electrical resistance and mechanical friction, allowing trains to move at higher speeds without wasting energy[12].

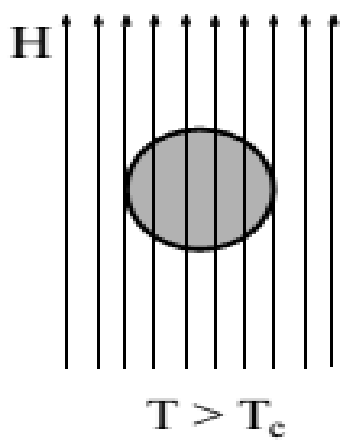

(a)

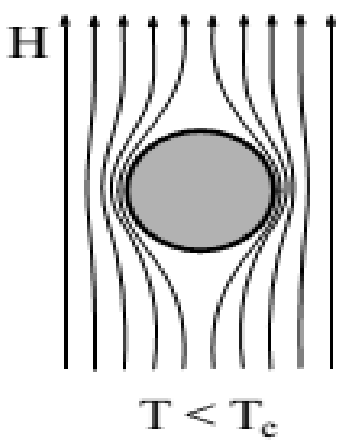

(b)

Figure 4: (a) Magnetic field lines penetrate through a superconductor at a temperature above its critical temperature $\left(T>T_{C}\right)$. (b) When the superconductor is cooled below its critical temperature $\left(T<T_{C}\right)$, magnetic field lines are expelled from the interior of the superconductor due to the Meissner effect[13][14] .

Two years later, in 1935 the London brothers, F. and H. London came up with a theory that explained the behavior of superconductors. They showed that the Meissner effect was a consequence of the minimization of the electromagnetic free energy carried by superconducting current. Their theory introduced many concepts that are still widely in use. For example, the London penetration depth $\lambda_{L}$ and the London equation, where $\lambda_{L}$ is an ideal theoretical limit that determines how deep into a bulk superconductor material a magnetic or an electric field can penetrate[15][16].

In 1950, Ginzburg-Landau theory of superconductivity was developed by Landau and Ginzburg. This theory, which combined Landau's theory of second-order phase transitions with a Schrödinger-like wave equation, had great success in explaining the macroscopic properties of superconductors. This theory speculated that the superconducting electrons were in a macroscopic quantum state and a macroscopic wave function (or order parameter) exists. It also provided a concept of the coherent length and a way to calculate the superconducting wave function. With the introduction of this theory, the classical period of superconductivity with Onnes, Meissner and the Londons came to an end, and the era of superconductivity as a macroscopic quantum phenomenon began. In particular, Abrikosov showed that Ginzburg-Landau theory predicts the division of superconductors into two categories now referred to as type I and type II superconductors. Abrikosov, Landau and Ginzburg were awarded the 2003 Nobel Prize in physics for their work (Landau having died in 1968)[17].

The full microscopic theory of superconductors was proposed in 1957 by Bardeen, Cooper, and Schrieffer. This BCS theory solved the many body problems for all the conduction electrons in a solid including the explanation of superconducting current as a superfluid of Cooper pairs, pairs of electrons interacting through the exchange of phonons. For this work, the authors were awarded the Nobel Prize in 1972[18][19].

Before 1986, the critical temperature, $T_{C}=23.2 \mathrm{~K}$ for the compound $\mathrm{Nb}_{3} \mathrm{Ge}$ was considered to be the maximum attainable value. However, in 1986 Bednorz and Mueller prepared a sample containing lanthanumbased cuprate (copper oxide), ( $\mathrm{BaLaCuO})$ materials and cooled them to low temperatures and discovered superconductivity in this material with a critical temperature of $35 \mathrm{~K}$ which opened up research for high temperature superconductors. Shortly, in the early 1987 by replacing the lanthanum with yttrium, i.e. making YBCO they obtained the critical temperature for this compound as high as $92 \mathrm{~K}$. This critical temperature hits a record value of $138 \mathrm{~K}$ by 1995 for the compound $\mathrm{HgBaCaCuO}[20][21][22]$. 


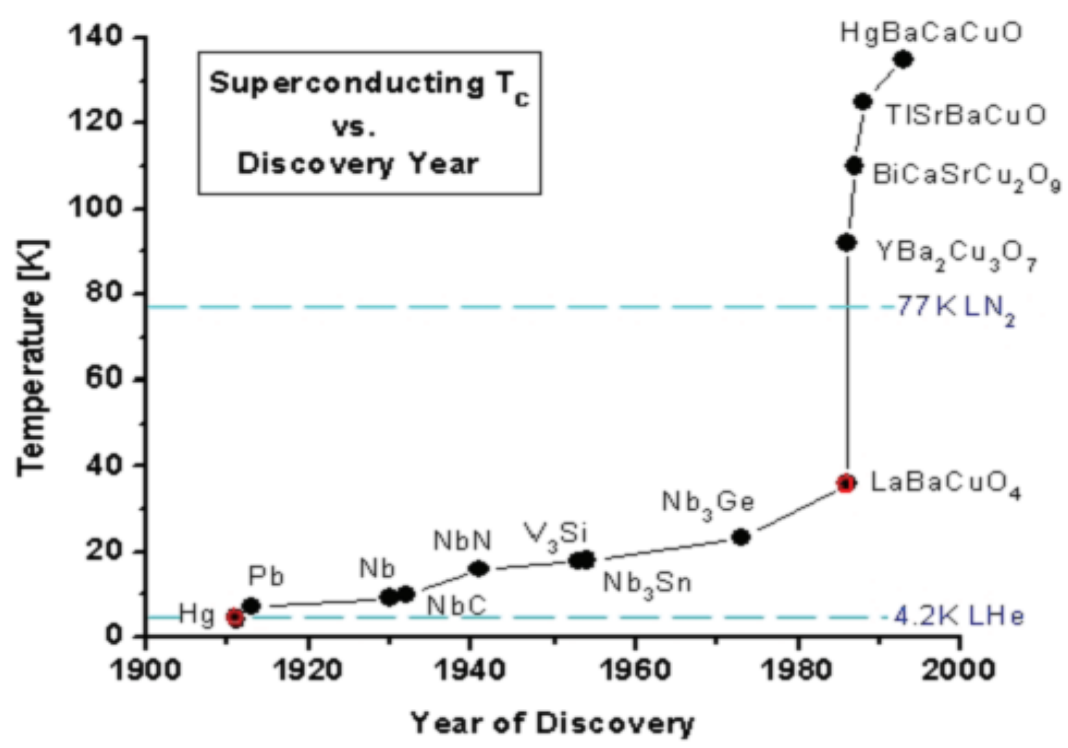

Figure 5: Superconducting $T_{C}$ vs. Discovery Year[17].

\section{TYPES OF SUPERCONDUCTORS}

Depending on their behavior in an external magnetic field, superconductors are divided into two parts. These are type I and type II superconductors.

Type I superconductors: - These are superconductors which lose superconductivity very easily or abruptly when placed in the external magnetic field. They also known as soft superconductors because of the reason that they lose their superconductivity easily. Figure 6a below shows intensity of magnetization $(M)$ versus applied magnetic field $(H)$, when the type I superconductors are placed in the magnetic field; they suddenly or easily lose their superconductivity at critical magnetic field $\left(H_{c}\right)$, point $\mathrm{A}[23]$.

For the value of magnetic field greater than critical magnetic field $\left(H_{c}\right)$, type I superconductors become normal conductors. These types of superconductors perfectly obey Meissner effect. Pure specimens of many materials (as an example Aluminum $\left(H_{c}=0.0105\right.$ Tesla $)$ and Zinc $\left(H_{c}=0.0054\right.$ Tesla $)$ ) are type I superconductors. Type I superconductors typically have critical temperatures ranging from less than $1 \mathrm{~K}$ to around $20 \mathrm{~K}$. Solid mercury, for example has a critical temperature of $4.2 \mathrm{k}[24]$.

Type II superconductors:-These are superconductors which lose their superconductivity gradually but not easily or abruptly when placed in the external magnetic field. Figure $6 \mathrm{~b}$ below shows intensity of magnetization $(M)$ versus applied magnetic field $(H)$, when the type II superconductors are placed in the magnetic field; they gradually lose their superconductivity starting from lower critical magnetic field $\left(H_{c 1}\right)$ and completely lose their superconductivity at upper critical magnetic field $\left(H_{c 2}\right)[25]$.

The region between the lower critical magnetic field $\left(H_{c 1}\right)$ and the upper critical magnetic field $\left(H_{c 2}\right)$ is known as the vortex or intermediate state. For the region $H>H_{c 2}$, the type II superconductors become normal conductor. These type II superconductors are also known as hard superconductors because of the reason that they lose their superconductivity gradually but not easily. They obey Meissner effect partially. Example of Type II superconductors: $\mathrm{NbN}\left(H_{c}=8 \times 10^{6}\right.$ Tesla $), \mathrm{Babi}_{3}\left(H_{c}=59 \times 10^{3}\right.$ Tesla). [26][27]. 


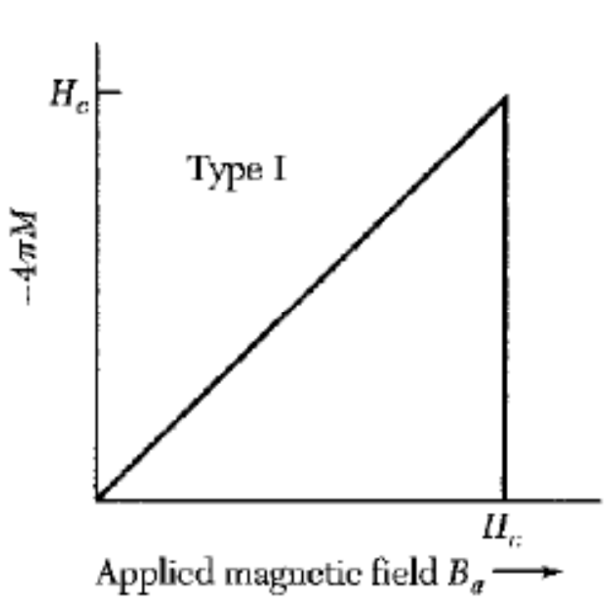

(a)

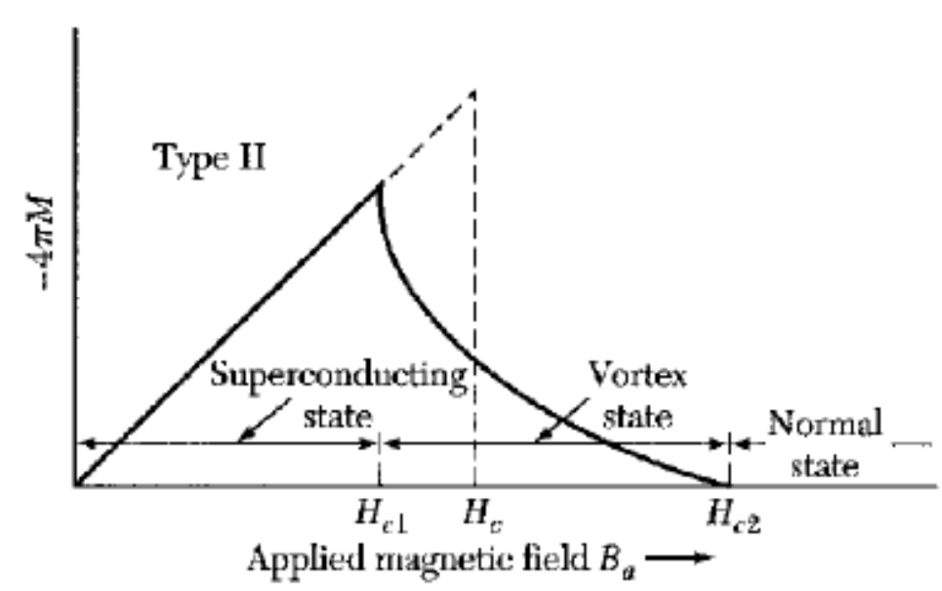

(b)

Figure 6: Types of superconductors[28].

\section{IMPORTANCE OF EXCEPTIONAL PROPERTIES OF SUPERCONDUCTORS}

Free electrons (lossless current with zero resistance) move through them with high current density plays an important role in transmission of electric power in superconductors that differentiate them from all conventional conductors. This property also enables more powerful magnets for motors, energy storage, generators, medical equipment and industrial separations[29]. The technology of superconductors is also attractive compared to conventional technology because superconductors are smaller, lighter and more efficient than ordinary conductors with permission of improvements in some cases. As superconductors have zero resistance accompanied with high frequencies and extremely low signal dispersion, they can be used in microwave components, communication technology and several military applications. Furthermore, superconductors have high sensitivity to magnetic field which allows them to provide a distinctive sensing capability thousand times superior to today's best conventional measurement technology[30].

\section{APPLICATIONS}

The property of zero resistance at low temperatures to the flow of electricity enables a wide range of innovation technology applications. Products from Superconductors are exceedingly environmentally friendly compared to their conventional counterparts. They generate no greenhouse gases and are cooled by non-flammable liquid nitrogen (nitrogen comprises $80 \%$ of our atmosphere) as opposed to conventional oil coolants that are both flammable and toxic. They are also typically at least 50\% smaller and lighter than equivalent conventional units which translate into economic incentives[31].

Therefore, due to the above mentioned exceptional properties, superconductors are used in wide variety of sectors including for power transmissions, magnets in generators, energy storage devices, medicine, high energy accelerator physics, levitated vehicle transportation, rotating machinery, magnetic separations, scientific research, industry, defense and for space mission[32]. In this paper only superconductor for electric power, transportation communication, medicine and defense is considered.

\section{Electric power}

Electric power system is one of the most important infra-structure for modern society's life style. Amount of electric energy, its continuity and the electric materials used for its storage, transmission and distribution to end users determines the level of development of a country. However, even in most highly developed electrical power system, there are several difficulties related from production, transmission and distribution. Usually, power generation is located remote area from the storage center, and thus long transmission and distribution lines have to be constructed to maintain uninterrupted and quality electric power for home based and industrial economic activities [33]. Therefore, reliable, cheap, efficient conductor is required for transmission and distribution of electric power. Most of the conductors used in modern power system facilities, for example, generator, transformer, transmission line, motor etc., are copper or aluminum made. These conductors have resistance, $\mathrm{R}$ which restricts the ability of thermal rating of electric facilities due to energy dissipate according to Ohm's law. If there is a conductor with no current loss, we can make efficient electrical facilities. Superconductors are a promising solution to make innovation on electric facilities because they have zero resistance. Wires made from superconductors have high power density and excellent electrical efficiency which results in highly compact, powerful devices and systems that are more reliable, efficient and friend to our environment[34][35]. 


\section{Transportation}

Transportation of people, goods and animals from one place to another by land, by sea and by air is a fundamental economic activity to sustain the life of human beings on our planet, earth. Modern transportation ways (including automobile, train, airplane and ship) had changed this sector dramatically by minimizing the time taken and increasing the number people as well as animal and amount of goods to large extent and it is always going under improvement. Until today the main problem of these transportation ways is their dependence on non-renewable energy sources which are not environmentally friendly[36][37]. Therefore, new technologies with excellent efficient and high speed accompanied with environmentally friendly manner must be developed. Because of their zero resistance and Meissner effect (the expulsion of a weak, external magnetic field from the interior of a superconductor) properties, superconductors have the ability to solve problems of environmental, efficient and speed challenges. Technologies from superconductors include ship propulsion systems, magnetically levitated trains and railway traction transformers. For example, a train can be levitated above its track using powerful, superconducting magnets, so that there is little friction[38].

\section{Communication}

Superconductors present the unique advantages of ultra-low dissipation and distortion as well as intrinsic (quantum) accuracy. These advantages enable them to give the most significant change to satellite communications in recent decades around the worldwide. Advanced filters are already deployed in commercial wireless base stations, enabling wider range and fewer dropped calls for wireless satellite communication. A more significant development involves a complete transition from analog to all-digital receivers and transmitters, a migration currently in the prototype phase which promises dramatic improvements in efficiency and cost for both military and commercial wireless communication systems[39].

\section{Medicine}

Due to their unique properties of having high-field magnets, with high current density and zero DC resistance, currently superconductors are widely in use in biomedical applications such as the Magnetic Resonance Imaging (MRI).Over the past decades, the extraordinary development of the cooling systems made it possible the introduction of superconducting magnets in MRI and enabled impressive improvements in the image resolution for medical diagnosis. MRI is currently the largest market for low temperature superconductor (LTS) wires while LTS magnets are the dominant technology for MRI. Advances in high temperature superconductors (HTS) promise more compact and less costly MRI systems with superior imaging capabilities. In addition, MagnetoEncephalography (MEG), Magnetic Source Imaging (MSI) and Magneto Cardiology (MCG) enable non-invasive diagnosis of brain and heart functionality[40][41].

\section{Defense}

The phenomenon of superconductivity brings sensitivity, accuracy and performance advantages beyond the theoretical limits of conventional electronics technology. In addition, using superconductors gives the benefit of size and weight reduction of $50 \%-70 \%$ when compared to conventional equipment[42]. Due to these unique properties superconductors have the following applications in defense (military):

- Body of ships build from superconductors help ships when they move on water body to develop a low level magnetic charge against magnetic field of the earth (demagnetizing magnetic field of the earth) which enables them to come back home after travelling long distances. Body of guided-missile destroyers can also be modified by high Temperature Superconductor (HTS) coils and this was tested by USA in 2008 and has performed extremely well in sea trials since that time[43].

- High Temperature Superconducting (HTS) power cables have significantly higher power density capability than copper counterparts, thus providing weight and size savings and making room for other war fighting equipment on board Navy vessels[44].

- Superconductor electronics can considerably enhance anti-ship missile defense radars. Emerging threats include sea-skimming missiles that reflect very small fractions of the radar signal. The challenges to radar receivers are that they must distinguish these small echoes from the huge background clutter of waves, rain, jammers and mountains on the shore in real time. Superconductivity enables the highest dynamic range digitizers and the smallest digits are essential to detect the most elusive threats. High dynamic range superconductor-based electronics provide the most advanced technology and simplify the receiver, thereby making these life-saving sensors and detectors affordable for a wide variety of Navy ships[45].

\section{CONCLUSION}

The phenomenon of superconductivity is characterized by three hallmark properties, these being zero DC resistance, a fully diamagnetic Meissner effect, and macroscopic quantum phenomena. These three main properties have been discovered in separate times. The zero DC resistance property was first discovered by Kamerlingh 
Onnes in 1911, and has since led to many important applications of superconductors in power transmission and energy storage. The second property is a spontaneous and essentially complete diamagnetic response developed by superconductors in the presence of a static magnetic field. As the material enters the superconducting state it will develop currents to exclude magnetic field from its interior. This phenomenon is known as the Meissner effect, and distinguishes superconductors from perfect conductors (which would not show a spontaneous Meissner effect).This second property has applications ranging from transportation to military and space mission. The third property which is called macroscopic quantum effects arises from the quantum mechanical nature of the superconducting correlated electron state. As discussed in this paper, materials that have the phenomenon of superconductivity are termed as superconductors and they are divided into two types depending on their behavior in an external magnetic field, these being type I and type II superconductors. Type I superconductors lose their superconductivity very easily or abruptly when placed in the external magnetic field while Type II superconductors lose their superconductivity gradually but not easily or abruptly when placed in the external magnetic field. Superconductors also divided into two types depending on the value of their critical temperatures. These are low and high temperature superconductors. Superconductors have wide range of applications ranging from high energy accelerator physics, energy, transportation, military to space mission.

\section{REFERENCES}

[1] M. A. Malik and B. A. Malik, "High Temperature Superconductivity: Materials , Mechanism and Applications," vol. 41, pp. 305-314, 2014.

[2] M. Atikur Rahman, "A Review on Cuprate Based Superconducting Materials Including Characteristics and Applications," Am. J. Phys. Appl., vol. 3, no. 2, p. 39, 2015.

[3] R. W. Simon, R. B. Hammond, S. J. Berkowitz, and B. A. Willemsen, "Superconducting microwave filter systems for cellular telephone base stations," Proc. IEEE, vol. 92, no. 10, pp. 1585-1595, 2004.

[4] L. Lemberger, "Vortex lattice in conventional and unconventional superconductors," no. January, 2016.

[5] C. P. Strehlow and M. C. Sullivan, "A classroom demonstration of levitation and suspension of a superconductor over a magnetic track," Am. J. Phys., vol. 77, no. 9, pp. 847-851, 2009.

[6] N. Murugan, M. N. Nejaamtheen, and S. S. Rajan, "Conceptual Design of an Aircraft with Maglev Landing System," vol. 7, no. 7, pp. 1603-1605, 2013.

[7] A. Abdallah Ahmed Elfaki, "The Effect of Temperature on Conductivity of Conductors and Superconductors," Am. J. Phys. Appl., vol. 5, no. 1, p. 1, 2017.

[8] J. R. Kirtley, "Fundamental studies of superconductors using scanning magnetic imaging," Reports Prog. Phys., vol. 73, no. 12, 2010.

[9] M. N. Wilson, “A century of superconducting technology,” AIP Conf. Proc., vol. 1435, no. June 2012, pp. 11-35, 2012.

[10] R. V Holla, "Energy Storage Methods - Superconducting Magnetic Energy Storage - A Review," J. Undergrad. Res. 5, vol. 1, 2015.

[11] mit, "Superconductivity : The Meissner Effect, Persistent Currents and the Josephson Effects," pp. 1-16, 2011.

[12] H. Yaghoubi and Hamid, “The Most Important Maglev Applications," J. Eng., vol. 2013, pp. 1-19, 2013.

[13] S. Fujita, S. Fujita, and S. Godoy, Theory of high temperature superconductivity. 2001.

[14] A. S. Gill, "Meissner Effect ( 1933 ) Re-Explained by Gill 's Electronic Theory of Magnetism ( 1964 )," vol. 4, no. 1, pp. 1-14, 1964.

[15] K. P. Dahal, "Superconductivity: A Centenary Celebration,” pp. 26-34, 1911.

[16] D. Rohner et al., "Real-space probing of the local magnetic response of thin-film superconductors using single spin magnetometry," Sensors (Switzerland), vol. 18, no. 11, 2018.

[17] A. Kruijt and M. Turin, "Review Article," Lang. Soc., vol. 46, no. 02, pp. 257-269, 2017.

[18] A. C. Rowat, "D Iversifying P Hysics E Ducation for," Phys. Canada, vol. 67, no. 2, pp. 103-106, 2005.

[19] T. M. Silver, "Holzindustrie wird Holzernter," Holz - Kurier, vol. 56, no. 17, p. 7, 2001.

[20] V. L. Ginzburg, "High-temperature superconductivity (history and general review)," Sov. Phys. Uspekhi, vol. 34, no. 4, pp. 283-288, 1991.

[21] S. Georgieva, T. Nedeltcheva, and A. Stoyanova- Ivanova, "Development of the Titrimetric and Spectrophotometric Methods for Determination of the Oxygen Content in Superconducting Cuprates," Am. Chem. Sci. J., vol. 13, no. 1, pp. 1-15, 2016.

[22] M. Başoğlu, "The effect of sintering in different atmospheres on superconductivity parameters of YBCO," Turkish J. Phys., vol. 41, no. 2, pp. 91-94, 2017.

[23] A. Nie and M. Williams, "Determination of the critical field and critical temperature for various Type I and Type II metals and alloys," vol. 02138, 2016.

[24] R. L. Fagaly, "Superconducting quantum interference device instruments and applications," Rev. Sci. Instrum., vol. 77, no. 10, pp. 1-45, 2006. 
[25] L. F. Cohen and H. J. Jensen, "Open questions in the magnetic behaviour of high-temperature superconductors - Abstract - Reports on Progress in Physics - IOPscience,” vol. 60, pp. 1581-1672, 1997.

[26] V. V. Moshchalkov et al., "Type-1.5 Superconductors," vol. 117001, no. March, pp. 1-4, 2009.

[27] E H Brandt, "The flux-line lattice in superconductors," Reports Prog. Phys., vol. 58, no. 11, p. 1465, 1995.

[28] P. Chaddah, "Critical current densities in superconducting materials," Sadhana - Acad. Proc. Eng. Sci., vol. 28, no. 1-2, pp. 273-282, 2003.

[29] “S UPERCONDUCTIVITY : ADVANCES AND PROSPECTS,” vol. 67, no. 2, p. 2011, 2011.

[30] H. Kamerlingh, "Elementary properties of superconductors," pp. 1-5, 1986.

[31] M Asghar et al., "Current and Future Prospecs of High Temperature Superconducting Products," vol. 54, pp. 27-36, 2014.

[32] S. K. Gupta, H. Jangam, and N. Sharma, "Review of High Temperature Superconductors and Application in Various Fields," pp. 1-9, 2018.

[33] T. Janowski et al., "Superconducting devices for power engineering," Acta Phys. Pol. A, vol. 130, no. 2 , pp. 537-544, 2016.

[34] B. Vaishnavi, R. A. Suji, ... D. T.-I. J. of, and U. 2016, "Superconducting fault current limiter \& its application," Ijser.Org, vol. 7, no. 5, 2016.

[35] M. M. Josephine, “9Dae127533C7a031228163F072E2E9556Ada,” vol. I, 2015.

[36] W. M. Shibani, M. F. Zulkafli, and B. Basuno, "Methods of Transport Technologies: A Review on Using Tube/Tunnel Systems," IOP Conf. Ser. Mater. Sci. Eng., vol. 160, no. 1, 2016.

[37] D. Pojani and D. Stead, "Sustainable urban transport in the developing world: Beyond megacities," Sustain., vol. 7, no. 6, pp. 7784-7805, 2015.

[38] J. H. Durrell et al., "Bulk superconductors: A roadmap to applications," Supercond. Sci. Technol., vol. 31, no. 10, 2018.

[39] S. K. Kolli, S. Velpula, and K. K. Yenubari, "Superconductors in the Field of Communication and Study Various Electric Properties,” vol. 6, no. 7, pp. 2015-2018, 2017.

[40] T. C. Cosmus and M. Parizh, "Advances in whole-body MRI magnets," IEEE Trans. Appl. Supercond., vol. 21, no. 3 PART 2, pp. 2104-2109, 2011.

[41] S. Saleem, "A Perspective on Medical Applications of High Temperature Superconductors," J. Bioeng. Biomed. Sci., vol. 04, no. 01, pp. 10-11, 2014.

[42] T. D. Industrial, T. Base, P. Microwave, M. Components, W. Analog, and H. P. Motors, "Military system applications of superconductors," vol. 15, no. 50, pp. 15-19, 1988

[43] E. L. I. Brookner, “ADVANCES AND RADARs AND PHASED- ARRAYs,” no. D1, pp. 781-862, 2017.

[44] D. U. Gubser and W. Dc, "US Navy's Superconductivity Programs Scientific Curiosity to Fleet Utility," vol. 935, no. 3, pp. 931-935, 2011.

[45] S. Denis et al., "Magnetic shielding properties of high-temperature superconducting tubes subjected to axial fields,” Supercond. Sci. Technol., vol. 20, no. 3, pp. 192-201, 2007. 